\title{
基于搜索空间缩减策略的供水管网抗震设计参数 优化
}

\author{
侯本伟，杜修力* \\ 北京工业大学城市与工程安全减灾教育部重点实验室, 北京 100124 \\ *E-mail: duxiuli@bjut.edu.cn
}

收稿日期: 2014-07-02; 接受日期: 2015-03-26

国家自然科学基金(批准号: 51421005, 51278017, 51208017)和教育部创新团队发展计划(批准号: IRT13044)资助项目

\begin{abstract}
摘要供水管网抗震优化设计模型以用户节点的抗震安全能力为设计目标, 包含管网拓 扑布局及管段结构抗震能力两个设计参数, 寻找最优设计参数是离散变量组合优化问题. 提出了一种基于两阶段搜索空间缩减策略的管网抗震优化设计模型求解方法，第一阶段利 用度约束连通图生成初始种群，缩小优化初始搜索空间；第二阶段采用违约个体修补策略 转换优化进行过程中不满足约束条件个体所处的搜索空间, 实现了优化过程中搜索空间的 动态缩减，提高了优化搜索的效率。此方法在供水管网抗震优化设计中有较高的效率，也可 为其他有约束离散变量优化问题提供参考.
\end{abstract}

关键词

供水管网

抗震优化设计

离散变量优化

搜索空间缩减

\section{1 引言}

城市遭受地震作用后, 基础设施网络系统的安 全运行能力直接影响震后救援和恢复的进度 ${ }^{[1]}$. 供水 管网系统是重要的城市生命线工程, 提升其抗震安 全性可通过提高管段单元的抗震能力和增强管网拓 扑圥余设置两种方法实现 ${ }^{[2]}$. 与供水管网日常功能优 化设计的方法类似, 管网的抗震设计参数可通过优 化求解得到 ${ }^{[3,4]}$. 供水管网抗震优化设计模型中, 以 管网最低造价为优化目标, 以用户节点的抗震连通 可靠性或功能可靠性为约束条件 ${ }^{[5]}$. 优化设计模型为 离散变量组合优化问题, 当网络规模和设计参数较 多时, 优化模型搜索空间较大, 提高优化计算的效率 是工程应用面临的重要问题.

在管网优化设计模型求解方面, Kadu 等人 ${ }^{[6]}$ 根据
模型特点分析及工程经验, 缩减了部分变量的编码 空间; 在管网优化算法研究方面, Tolson 等人 ${ }^{[7]}$ 、庄宝 玉等人 ${ }^{[8]}$ 分别采用遗传算法、蛙跳算法求解管网日常 功能优化设计模型; 刘威等人 ${ }^{[9]}$ 比较了遗传算法、遗 传一模拟退火算法、蚁群算法和微粒群算法在求解管 网抗震拓扑优化问题的适用性. 这些比较工作并未 根据管网优化设计模型的特点对算法本身进行改进.

标准遗传算法(GA)中，初始种群多采取随机生 成方法得到, 然而初始种群对于算法的收玫速度有 很大影响 ${ }^{[10]}$. 为保持初始种群多样性信息并获取较 优的初始种群个体, Leung 和 Wang ${ }^{[11]}$ 、何大阔等人 ${ }^{[12]}$ 分别采用变量定义空间的正交设计、均匀设计方法产 生非随机初始种群, 张建勇和李军 ${ }^{[13]}$ 提出了特定问 题的初始种群生成改进方法.

采用 GA 求解有约束优化问题时，约束条件的处

引用格式: 侯本伟, 杜修力. 基于搜索空间缩减策略的供水管网抗震设计参数优化. 中国科学: 技术科学, 2015, 45: 747-756 Hou B W, Du X L. A heuristic search space reduction method for water distribution system seismic design optimization (in Chinese). Sci Sin Tech, 2015, 45: 747-756, doi: 10.1360/N092014-00235 
理可采用罚函数法、违约个体修补法、多种群进化、 多目标模型转化等 ${ }^{[14 ~ 16]}$, 其中前两种方法为较为常 用. 在罚函数法中, 具体问题的罚值系数是影响优化 效率的关键因素. 在违约个体修补法中, 很难找到适 用各种问题的普适性修补方法算法, 尤其对于无法 用显式表达的约束条件，一般需要根据具体问题制 定修补策略 ${ }^{[3,17,18]}$, 个体修补产生的计算量可能远大 于进化过程本身的计算量.

本文以 GA 为基准, 提出了一种供水管网抗震优 化设计模型求解的两阶段搜索空间缩减方法. 第一 阶段利用度约束连通图(degree constrained connected graph, DCCG)生成初始种群代替随机种群, 缩小初 始优化搜索空间. 第二阶段采用个体修补和罚函数 相结合的约束条件处理方法, 通过修补策略转换优 化过程中不满足局部约束条件个体的所处空间, 实 现了优化过程中搜索空间的动态缩减. 此方法适用 于多个约束条件, 且不同约束条件计算量相差较大 的情况; 将约束条件按计算量分为较小计算量 (I 类) 和较大计算量(II 类)两类; 对 I 类违约个体进行个体 修补, 对 II 类违约个体进行罚函数处理, 从而避免修 补第 II 类约束条件造成的大量计算.

\section{2 管网抗震设计参数优化分配模型}

考虑管网觉余性和管段不同抗震能力的管网抗 震优化设计模型为

$$
\begin{aligned}
& \min C=\sum \gamma_{i j} \cdot c\left(l_{i j}, d_{i j}\right) \cdot \varphi_{i j}, \\
& \text { s.t. } 1) j \in\left\{T_{S \rightarrow j} ; j=1,2, \cdots, n\right\}, \\
& \text { 2) } R_{j} \geqslant\left[R_{j}\right], \\
& \text { 3) } P_{j} \geqslant\left[P_{j}\right],
\end{aligned}
$$

其中 $c\left(l_{i j}, d_{i j}\right)=\left(a_{1}+a_{2} \cdot d_{i j}^{a_{3}}\right) \cdot l_{i j}$ 为管段的造价, $l_{i j}, d_{i j}$ 分别 为节点 $i, j$ 之间的管段长度 $(\mathrm{m})$ 和直径 $(\mathrm{m}) ; \gamma_{i j}$ 为连通系 数, 铺设该管段取 1 , 不铺设则取 $0 ; a_{1}, a_{2}$ 和 $a_{3}$ 是管段 造价常数, 可以根据实际工程的造价拟合得到. $\varphi_{i j}$ 为 管段抗震能力造价调整系数, 管段抗震能力及相应 的 $\varphi_{i j}$ 与实际可选的管材及抗震措施有关. 约束(1)中 $T_{s \rightarrow j}$ 表示源点 $s$ 与节点 $j$ 连通, $n$ 为管网的用户节点总 数. 约束(2)中 $R_{j}$ 为节点连通㝋余度, 其取值与该点相 连的边数有关, 本节采用节点连接度表示; 约束(3)中 $P_{j}$ 为源点到用户节点的抗震连通可靠度, 可采用基 于宽度优先搜索(BFS)的 Monte Carlo 随机模拟 ${ }^{[4]}$ 求得;
$\left[P_{j}\right]$ 和 $\left[R_{j}\right]$ 分别为第 $j$ 节点的抗震连通可靠性和圥余度 约束.

针对以拓扑结构和管段抗震能力为设计参数的 管网抗震优化问题((1)式), 约束条件分别为: (1)连通 性约束, 可采用 1 次 BFS 搜索得到; (2)节点度约束, 可采用邻接矩阵的分列求和得到; (3)连通可靠性约 束, 采用基于 BFS 的 Monte Carlo 模拟法求解时, 需 进行 2000 次以上抽样, 每次抽样均需采用 BFS 搜索 判别连通状态. 由上述分析看出, 约束(1)(2)的校核计 算量较小(I 类), 约束(3)校核的计算量最大(II 类). 采 用 GA 算法求解式(1)时, 对违反约束(1)(2)的个体采用 修补方法, 对违反约束(3)的个体采用罚值处理.

\section{3 编码空间与约束条件处理}

采用 $\mathrm{GA}$ 算法求解(1)式优化模型, 用整数编码 表示个体, 1 个整数表示 1 条管段, 其数值代表了管 段的抗震能力. 例如, 当某管段的可选抗震能力有 3 种时, 其对应基因串的整数值可取 $0,1,2,3$; 取值 $1 \sim 3$ 代表管段铺设且抗震能力为相应的数值对应的 等级, 取值为 0 时表示管段不铺设. 所有备选管段的 基因串按管段编号排列在一起构成一条染色体; 一 条染色体代表一种管网设计方案. 当管段数量为 $m$, 每个管段有 $k$ 种可选抗震等级时, 编码的搜索空间 $\boldsymbol{\Omega}$ 为 $(k+1)^{m}$.

由于约束条件的存在导致编码空间 $\boldsymbol{\Omega}$ 大于可行 解空间 $\boldsymbol{\Omega}^{c}$ (同时满足所有约束条件的解空间), 如图 1 所示. (1)式模型的编码空间、约束空间、解空间关系 见图 2. 由于验算约束(1)和(2)的计算量较小, 先采用 违约个体修补方法将搜索空间限定于满足约束 (1) 和 (2)的局部空间 $\boldsymbol{\Omega}^{\prime \prime}\left(\boldsymbol{\Omega}^{c} \leqslant \boldsymbol{\Omega}^{\prime \prime} \leqslant \boldsymbol{\Omega}\right)$, 再进行满足约束(3)的 可行解空间搜索 $\left(\boldsymbol{\Omega}^{\prime \prime} \rightarrow \boldsymbol{\Omega}^{c}\right)$, 如图 3 所示. 本节采用

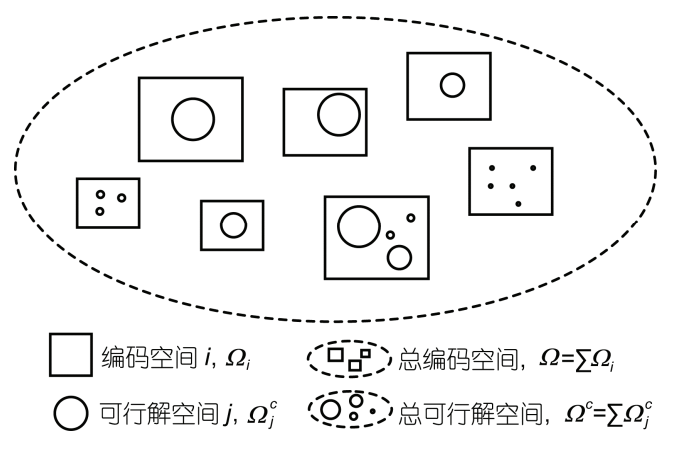

图 1 优化模型编码空间和解空间示意 



图 2 约束空间关系示意

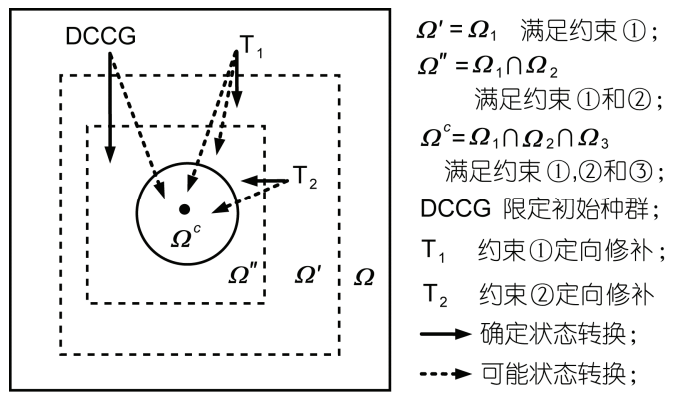

图 3 搜索空间缩减策略示意

“DCCG”生成初始搜索空间，利用“约束指导个体修 补策略”修补优化过程生成的违背约束 (1), (2)的个体, 将所有个体限定于局部空间 $\boldsymbol{\Omega}^{\prime \prime}$ 内, 采用罚函数法处 理位于 $\boldsymbol{\Omega}^{\prime \prime}-\boldsymbol{\Omega}^{c}$ 空间内的个体.

\section{1 “DCCG”生成初始种群}

约束(1)要求所有用户节点与源点全连通, 约束 (2)要求节点的圥余度达到限定要求; 约束 (1)、(2)的要 求和 “度约束最小生成树” 的约束条件相似, 二者区 别在于对节点度限定值的要求分别为大于和小于关 系. 这里将满足约束(1)、(2)要求的网络拓扑结构称为 “DCCG”, 提出基于 DCCG 生成初始种群拓扑结构 的方法; 与随机产生初始种群相比, 此方法实际缩小 了优化模型的初始搜索空间. 与式(1)优化模型的目 标函数在 $\varphi_{i j}=1.0$ 及约束条件(1)、(2)一致, 求解满足全 连通和度约束的总权值最小的网络拓扑结构. DCCG 问题的模型为

$$
\begin{aligned}
& \min Z=\sum_{i=1}^{n} \sum_{j=1}^{n} w_{i j} \gamma_{i j}, \\
& \text { s.t. } 1) j \in\left\{T_{S \rightarrow j} ; j=1,2, \cdots, n\right\}, \\
& \text { 2) } \sum_{i=1}^{n} \gamma_{i j} \geqslant\left[R_{i}\right],
\end{aligned}
$$

其中 $\boldsymbol{W}=\left(w_{i j}\right)_{n \times n}$ 为与 $G$ 的邻接矩阵 $\boldsymbol{A}$ 对应的边权(管 段造价)矩阵, $w_{i j}=c\left(l_{i j}, d_{i j}\right)$ 为管段的权重对应基本抗震 能力时 $\left(\varphi_{i j}=1.0\right)$ 的造价，当节点 $i$ 与 $j$ 间无连接管段时, $w_{i j}=\infty$.

度约束生成树问题的基本模型是 NP 难问题 ${ }^{[19]}$, 基于 GA 的优化搜索方法可以较好的解决这一问 题 ${ }^{[20]}$. 本节是为得到满足(2)式约束条件的可行解集, 并以此当作(1)式的初始解, 而非只求出一个满足约 束要求的最优解, 因此对最优解的求解并无严格限 定. 参考度约束生成树问题的解法, 可采用基于编码 空间搜索的 GA 求解 DCCG 问题模型.

此模型中每个管段有 2 种可选属性, 采用 01 编 码表示个体, 当管段铺设时取值 1 , 不铺设时取值 0 , 编码的搜索空间为 $2^{m}$. 采用 $\mathrm{GA}$ 求解的最终结果中所 有满足约束条件的个体 (并不要求最优个体)均可作 为(1)式初始种群备选个体. (2)式模型采用 01 编码, 只考虑了管网拓扑结构, 并未考虑管段抗震能力变 化; 由(2)式的可行解集变换为(1)式初始种群时, 有 以下两种处理方式: 1) 直接将(2)式可行解当作(1)式 初始解, 这里称之为“DCCG 01 种群”; 2) 对(2)式可 行解个体编码进行调整, 将个体中数值为 1 的基因位 用 $\operatorname{int}\{\operatorname{rand}\{1, k\}\}$ 值替换, 其中 $\operatorname{rand}\{*\}$ 在给定区间内 产生随机数, $\operatorname{int}\{*\}$ 为取整操作, 这里称之为“DCCG 随机种群”.

\section{2 违约个体修补策略}

在 GA 进化过程中, 交叉操作和变异操作生成的 新种群中存在不满足约束(1), (2)的个体, 本节采用分 别针对约束(1), (2)的定向修补策略进行“搜索空间变 换”.

\subsection{1 基本定义}

假定备选管网的网络结构 $G(\boldsymbol{V}, \boldsymbol{E})$ 由顶点(用户) 集合 $\boldsymbol{V}$ 和边(管线)集合 $\boldsymbol{E}$ 组成，其中 $\boldsymbol{V}=\left\{v_{1}, v_{2}, \ldots, v_{n}\right\}$, $\boldsymbol{E}=\left\{e_{1}, e_{2}, \ldots, e_{m}\right\}, n$ 和 $m$ 分别为 $G$ 的节点数和边数, 网 络源点 $s \in \boldsymbol{V}$. 对 $\forall v_{i}\left(v_{i} \in \boldsymbol{V}, v_{i} \neq s\right)$ 定义: 节点连接度 $d_{i}$, 当前拓扑结构中节点 $i$ 连接的边数; 节点级数 $l_{i}$, 源点 $s$ 连接到节点 $i$ 所需的最少边数; 节点最短距 $u_{i}$, 考虑边长权重后, 源点 $s$ 连接到节点 $i$ 所需的最短长 度; 节点邻接点集 $\boldsymbol{V}_{i}{ }^{B}$, 表示初始备选网络中 $v_{i}$ 点连 接的点集; 个体节点可连线点集 $\boldsymbol{V}_{i}^{B C}$, 表示属于 $\boldsymbol{V}_{i}^{B}$ 且已与源点连通的点集. 
将不连通节点(源点与该节点之间无连通路径) 表示为 $v_{i}^{N}\left(l_{i}=\infty\right)$, 将不连通节点分为两类: 1$)$ 节点度 为 $0\left(d_{i}=0\right)$ 的节点 (孤立节点) $\left.v_{i}^{N 1} ; 2\right)$ 节点度大于 $0\left(d_{i}>0\right)$ 的节点 $v_{i}^{N 2}$ (存在多个不连通点, 不连通点之间 相连成不连通区域). 不通区域集 $\boldsymbol{S}=\left\{\boldsymbol{S}_{j}, j=1,2, \ldots,|\boldsymbol{S}|\right\}$, $|\boldsymbol{S}|$ 为集合 $\boldsymbol{S}$ 中元素的个数; 不连通区域 $\boldsymbol{S}_{j}=\left\{v_{i}^{N 2}, i=\right.$ $\left.1,2, \ldots,\left|\boldsymbol{S}_{j}\right|\right\}$.

\subsection{2 约束(1)定向修补}

违反约束(1)的个体, 存在不连通节点, 针对约束 (1)的修补又可称为个体连通性修补. 刘威等人 ${ }^{[9]}$ 对节 点度为 0 的节点进行修补, 以保证节点度均大于 0 , 但“节点度大于 0 ”是“节点连通”的必要而非充分条件, 因此节点度大于 0 并不能保证个体中所有节点的全 连通，按照该方法修补后的个体仍可能为不连通个 体(图 4, 修补策略 $\mathrm{T}_{1,1}$ ). 李杰和邢燕 ${ }^{[3]}$ 提出的自动修 补方法中, 对所有不连通节点均按照设定连线概率 $\left(P_{\mathrm{L}}\right)$ 进行增设可选邻接边. 由于 $P_{\mathrm{L}}$ 不易确定, 设置过 小时, 修补后个体仍可能为不连通个体; 设置过大会 导致修补后个体的网络拓扑结构过于稠密, 从而增 加个体的造价, 与(1)式优化模型的目标函数相悖; 这种稠密型个体修补方法与约束(2), (3)要求并不同, 此方法修补后个体中节点的咒余度、连通可靠度存在 盲目性(图 4, 修补策略 $\mathrm{T}_{1,2}$ ).

本节提出一种基于最短距优先个体修补策略(图 4 , 修补策略 $\mathrm{T}_{1,3}$ ), 根据模型约束条件定向指导个体 修补. 图 4 中用 $r^{1}$ 表示约束(1)修补后个体所在解空间 位置用与最优解位置的距离, $r^{1}$ 的数值可由修补后个 体的适应度值倒数(目标函数值+罚值)表示, $r^{1}$ 值越大 说明修补后个体适应度越小, 个体位置离最优解越 远. 图 4 中存在关系 $r_{1}{ }^{1}<r_{2}{ }^{1}$, 也即 $r_{1}{ }^{1}$ 表示图 4 中 3 种 约束(1)修补策略的最优修补方法对应的位置.

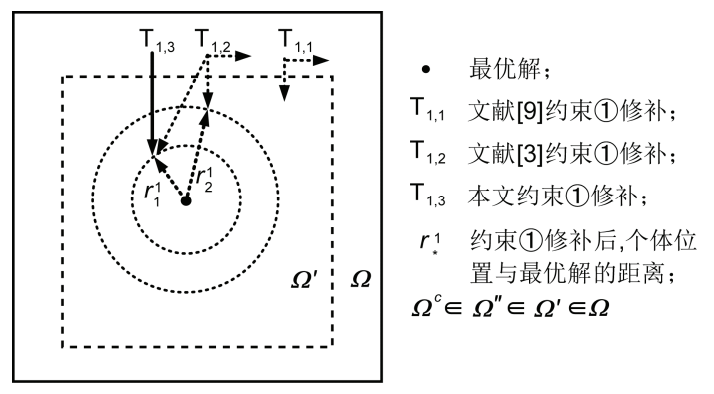

图 4 约束(1)不同修补策略比较
基于最短距优先的个体连通性修补策略：对第 1) 类不连通节点, 当有多个可连线点时, 只选取可使源 点与不连通点连接距离最短的 1 个可连线节点进行 连线; 对第 2) 类不连通节点区域, 只选取其中 1 个级 数最高的节点进行连线修补, 则该不连通节点集中 的全部节点变为连通节点. 具体步骤如下:

步骤 1 , 计算初始备选管网中所有节点的级数 $l_{i}$ 及源点最短距 $u_{i}$.

步骤 2, 找出不连通节点 $v_{i}^{N}$. 判别个体中所有节 点的连通性, 将不连通节点分为 $v_{i}^{N 1}$ 和 $v_{i}^{N 2}$ 两类, 找 出不连通区域集 $\boldsymbol{S}$.

步骤 3, 修补不连通节点. 对于 $v_{i}^{N 1}$, 在其邻接节 点集 $\boldsymbol{V}_{i}^{B}$ 中选出可连线点集 $\boldsymbol{V}_{i}^{B C}$, 当可连线点有多个 $\left(\left|\boldsymbol{V}_{i}^{B C}\right|>1\right)$ 时, 选择节点 $j\left\{j=\min \left\{u_{k}\right\}, k \in \boldsymbol{V}_{i}^{B C}\right\}$ 进行连线. 对由 $v_{i}^{N 2}$ 组成的每个不连通区域 $\boldsymbol{S}_{j}$, 选取节点 $h\left\{h=\min \left\{l_{k}\right\}, k \in S_{j}\right\}$ 作为此区域与外部连通节点的 “待 连线点”, 找出此 “待连线点” 的 $\boldsymbol{V}_{i}^{B C}$ 进行连线, 若 $\left|\boldsymbol{V}_{i}^{B C}\right|>1$, 选择节点 $j\left\{j=\min \left\{u_{k}\right\}, k \in \boldsymbol{V}_{i}^{B C}\right\}$ 进行连线.

步骤 4, 重复步骤 3 直至完成所有 $v_{i}^{N}$ 的修补.

\subsection{3 约束(2)定向修补}

对不满足度约束(约束(2)的节点, 从度约束最多 的点开始修补, 当有相同顺序时按级数优先原则; 按 源点距最小优先原则选择当前连线点的可连线点, 当可连线点中含有不满足度约束点时, 则优先选择 此类点为连线点. 具体步骤如下:

步骤 1 , 找出所有不满足节点度要求 $R_{i}<\left[R_{i}\right]$ 的节 点集 $\boldsymbol{V}^{D}\left\{v_{i}^{D}, i=1,2, \ldots,\left|\boldsymbol{V}^{D}\right|\right\}$.

步骤 2 , 当 $\left|\boldsymbol{V}^{D}\right|>1$ 时, 对所有 $v_{i}^{D}$ 先按 $\left[R_{i}\right]$ 降序排 列, 再对 $\boldsymbol{V}^{D}$ 按 $l_{i}$ 升序排列, 选取 $\boldsymbol{V}^{D}$ 序列顶部节点为 当前修补点 $v_{k}^{D}$.

步骤 3, 若 $R_{k}<\left[R_{k}\right]$, 对 $v_{k}^{D}$ 进行度连线修补, 在其 邻接节点集 $\boldsymbol{V}_{k}{ }^{B}$ 中选出可连线点集 $\boldsymbol{V}_{k}{ }^{B C}$; 当 $\left|\boldsymbol{V}_{k}{ }^{B C}\right|>1$ 时, 对所有可连线节点 $i \in \boldsymbol{V}_{k}^{B C}$ 按 $u_{i}$ 降序排列; 当存 在 $v_{i}{ }^{D} \in \boldsymbol{V}_{k}{ }^{B C}$ 时, 对 $\boldsymbol{V}_{k}{ }^{B C}$ 按 $\left[R_{i}\right]-R_{i}$ 降序排列; 选取 $\boldsymbol{V}_{k}{ }^{B C}$ 序列顶部节点 $j$ 为连线点; $R_{k}=R_{k}+1$; 若存在 $v_{i}{ }^{D} \in \boldsymbol{V}_{k}{ }^{B C}$, $R_{j}=R_{j}+1$.

步骤 4 , 重复步骤 3 , 直至完成当前修补点 $v_{k}^{D}$ 的 度修补。

步骤 5 , 重复步骤 1 4, 直至完成所有节点度修补. 违约个体在进行过约束(1)修补后所处的空间位 置与 $\boldsymbol{\Omega}^{\prime \prime}$ 的空间关系有两种可能, 如图 5 所示. 图 5(a) 


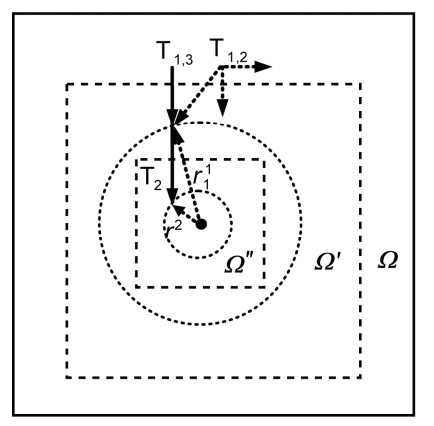

(a)
$\mathrm{T}_{2}$ 本文约束 (2)修补;

$r_{1}^{1}$ 采用较好的约束 (1) 修 补策略后, 个体位置与 最优解的距离 ;

$r^{2}$ 分别进行 约束 (1),(2)修 补后,个体位置 与最优 解的距离:

$\Omega^{\circ} \in \Omega^{\prime \prime} \in \Omega^{\prime} \in \Omega$

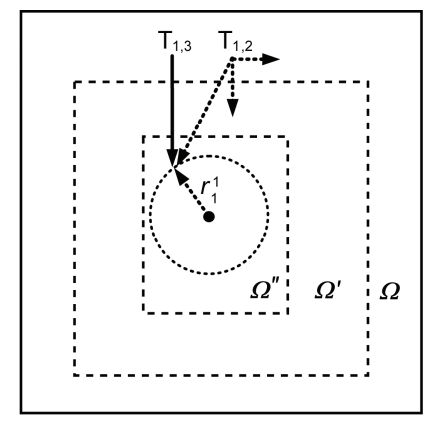

(b)

图 5 约束(2)修补策略示意

(a) 等待修补个体所处的解空间位置; (b) 无需修补个体的解空间位置

表示约束(1)修补后, 个体处于 $\boldsymbol{\Omega}^{\prime \prime}$ 外部, 因此需进行 约束(2)修补(图 5(a)中 $\mathrm{T}_{2}$ ), 进行过 $\mathrm{T}_{2}$ 修补后的个体与 最优解的距离为 $r^{2}$. 图 5(b)表示约束(1)修补后, 个体 已处于 $\boldsymbol{\Omega}^{\prime \prime}$ 内部, 则无需再进行约束(2)修补, 进行过 约束(2)校核后的个体与最优解的距离为 $r_{1}{ }^{1}$.

对比图 4 和 5 中的约束(1)和(2)的修补方法, 令 $r\left(\mathrm{~T}_{1,2}\right)=\left\{r_{1}{ }^{1}, r_{2}{ }^{1}\right\}, r\left(\mathrm{~T}_{1,3}\right)=\left\{r_{1}{ }^{1}\right\}, r\left(\mathrm{~T}_{1,3} \& \mathrm{~T}_{2}\right)=\left\{r_{1}{ }^{1}, r^{2}\right\}$ 分 别表示违约个体 $i$ 采用 $\mathrm{T}_{1,2}, \mathrm{~T}_{1,3}$ 和 $\mathrm{T}_{1,3} \& \mathrm{~T}_{2}$ 策略进行修 补后与最优解的距离. 对于仅存在连通性约束的个 体, 有 $r\left(\mathrm{~T}_{1,2}\right) \geqslant r\left(\mathrm{~T}_{1,3}\right)$; 对于同时含有连通性约束和午 余度约束的个体, 则有 $r\left(\mathrm{~T}_{1,2}\right) \geqslant r\left(\mathrm{~T}_{1,3} \& \mathrm{~T}_{2}\right)$. 由此看出, 本节提出的修补策略 $\left(\mathrm{T}_{1,3} \& \mathrm{~T}_{2}\right)$ 更为有效.

\section{3 个体修补示例}

选取文献[3]中的算例(图 6)说明本文修补方法与 文献[3]修补方法的区别. 备选管网中节点 1 为源点, 假定所有管段 $\left(e_{1} \sim e_{11}\right)$ 的长度均相同, 各节点的级数 如图 6(a)虚框中数字所示.

采用文献[3]修补方法, 以连线概率 $P_{\mathrm{L}}=1.0$ 对图 6(b) 个体进行连通修补的过程见图 7; 对于节点 4, 节

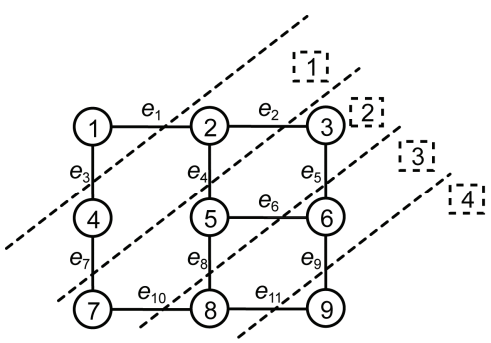

(a)

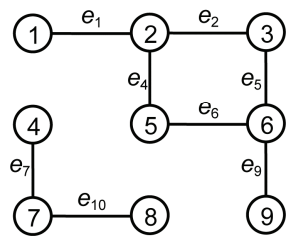

(b)
图 6 修补示例管网拓扑结构

(a) 初始备选管网; (b) 断开个体
点 1 为可连线点, 连线 $e_{3}$; 对于节点 7 , 邻接边均已存 在于当前拓扑中, 无法再连线; 对于节点 8 , 节点 5,9 为可连线点, 连线 $e_{8}, e_{11}$.

采用本文方法对图 6(b) 个体进行连通修补的过 程见图 8(a), (b); 断开节点 4, 7, 8 位于相同不连通区 域内(图 8(a)), 选取源点距和级数最小的节点 4 进行 连通修补(图 8(b)), 则节点 4, 7, 8 均成为连通节点; 对节点度 2 约束修补过程见图 8(c), 节点 8,9 均不满 足度约束, 按级数优先遍历节点 8 , 在节点 8 的可选 连线点(节点 5,9)中选择不满足度约束的节点 9 连线, 则节点 8,9 满足度 2 约束; 再遍历节点 9 , 也满足度 2 约束, 无需再进行修补.

\section{4 优化模型求解}

求解(1)式优化模型采用的 GA 算子设置如下:

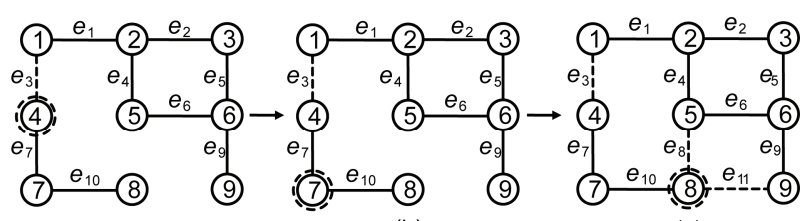

(a)

(b)

(c)

图 7 文献[3]连通修补过程

(a) 修补不连通点 4; (b) 遍历不连通点 7; (c) 修补不连通点 8

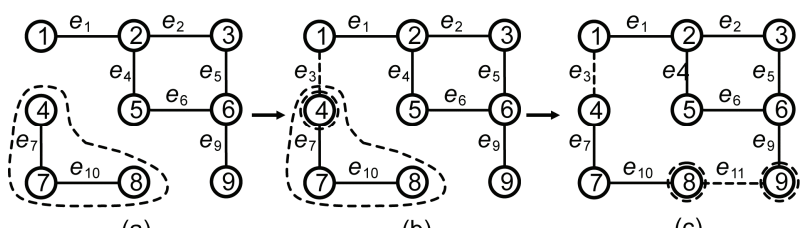

(a)

(b)

(c)

图 8 本文连通修补和几余修补过程 (a) 划分不连通区域; (b) 修补不连通节点; (c) 修补满足度约束点 
采用比例选择及最佳个体保留方法, 单点交叉, 基本 位变异, 最大进化代数收玫准则, 个体 $i$ 的适应度函 数定义见(3)式, 模型求解流程见图 9. 优化求解过程 中, 各代种群中个体所处的搜索空间变化如图 10 所示.

$$
\operatorname{Fit}(t)=C_{\text {scale }} /(\operatorname{Obj}(i)+\operatorname{pen}(i)) \text {. }
$$

其中 $C_{\text {scale }}$ 为正值调比因子; $\operatorname{Obj}(i)$ 为第 $i$ 个体的目标 函数值 (即造价 $C) ; \operatorname{Pen}(i)=w \cdot K \cdot(N-C(i))$ 为第 $i$ 个 体不满足约束条件(3)时的罚函数, $w=\max \left\{w_{i j}\right\}, K$ 为 罚值调整系数, $N$ 为含有约束条件(3)的节点个数, $C(i)$ 为与 $N$ 对应的个体 $i$ 满足约束条件(3)的节点个数. 需 要说明的是, 罚值调整系数 $K$ 的取值会影响优化算 法的求解效率：当取值过小时，会导致进化速度过慢，
进化代数过大; 当取值过大时, 会导致进化早熟的现 象. $K$ 的合理取值, 受到管网布局及目标函数公式等 多种因素的影响, 作者建议可以先进行小规模的试 算, 以得到合理的参考取值.

\section{5 算例分析}

选取文献[21]中的由 17 个用户节点和 27 条可选 供水管线构成的管网为例说明本文方法的有效性, 备选管网拓扑结构及管段属性见图 11, 节点 1 为源点, 其他节点为用户节点. 管段造价常数取值为 ${ }^{[9]}$ : $a_{1}=62.105 、 a_{2}=1979.7$ 和 $a_{3}=1.486$, 由此求得的造价

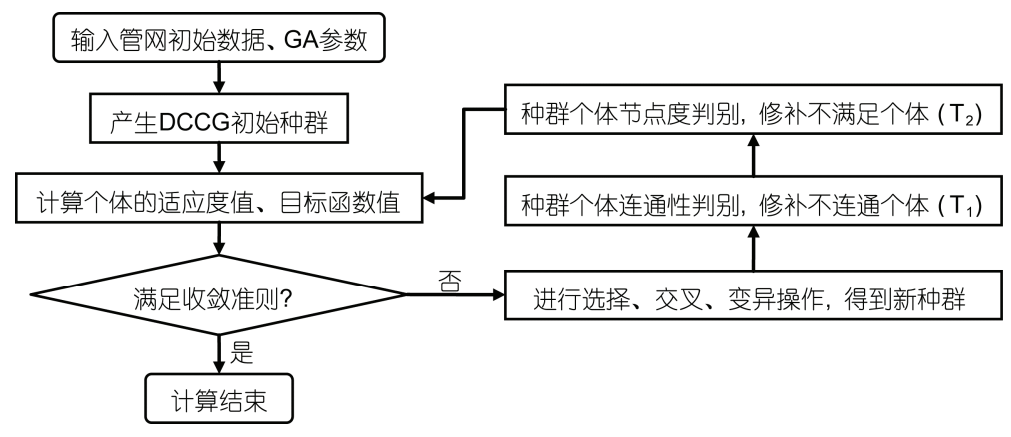

图 9 基于 $\mathbf{G A}$ 的管网抗震设计优化求解流程图

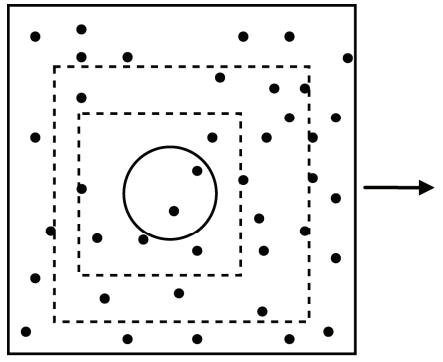

(a)

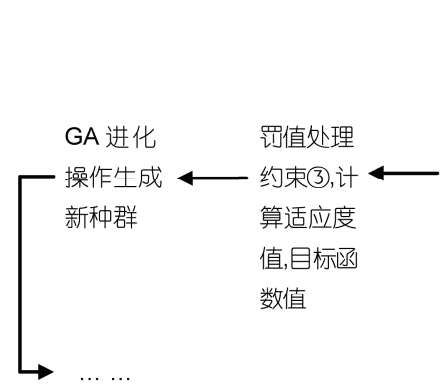

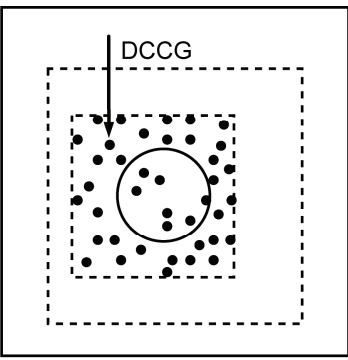

(b)

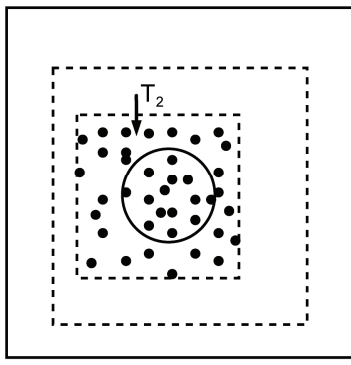

(e)

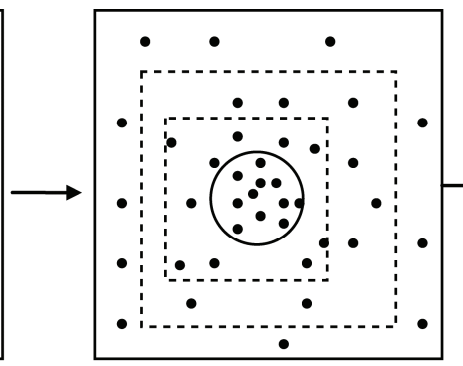

(c)

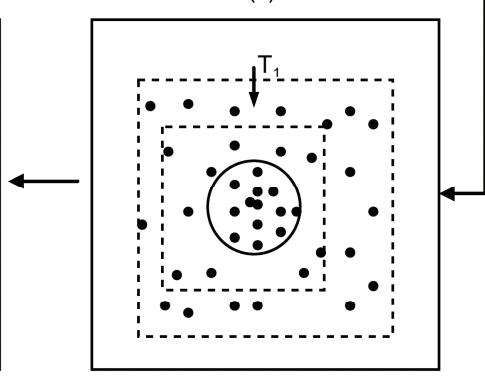

(d)

图 10 种群个体搜索空间分布

(a) 产生 $\boldsymbol{\Omega}$ 空间随机种群; (b) 生成 $\boldsymbol{\Omega}$ "空间初始种群; (c) GA 进化操作生成新种群; (d) 约束(1)定向修补种群; (e) 约束(2)定向修补种群 


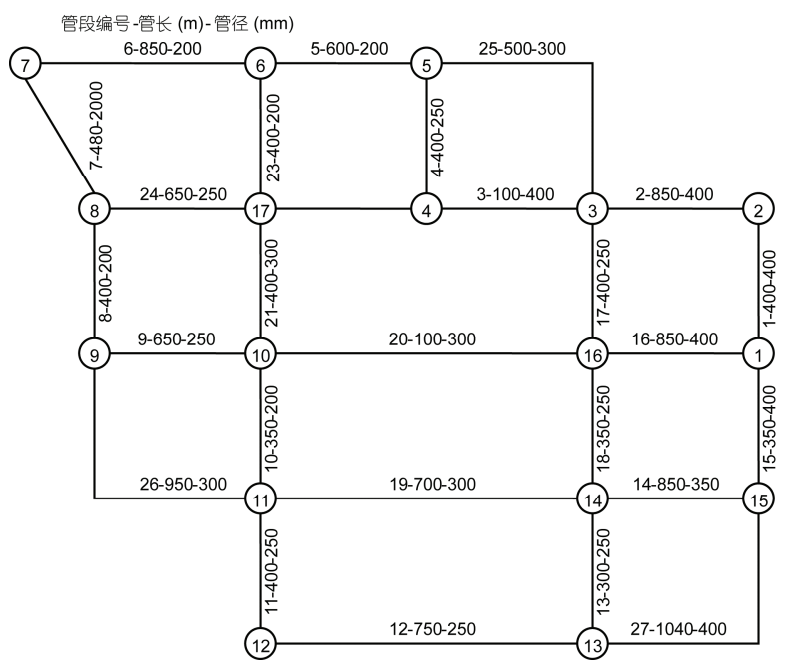

图 1117 点 27 边算例备选管网

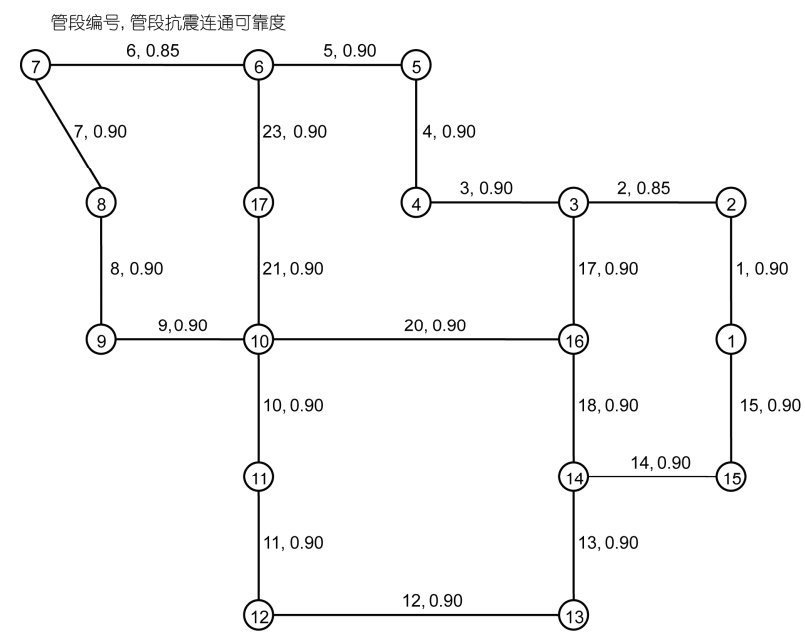

图 12 管网优化设计结果 $\left(3.2727 \times 10^{6}\right.$ 元 $)$

为基准造价 $(\varphi=1.0)$. 假定所有管段抗震性能分为 3 个 等级, 其抗震可靠度分别为 $0.90,0.85,0.95$, 相应的 造价调整系数 $(\varphi)$ 取值分别为 $1.0,0.9,1.2$, 与其对应 的 GA 个体编码为 $\{1,2,3\}$. 为保证节点连通可靠度 $\left(P_{j}\right)$ 计算的精度, Monte Carlo 模拟次数设定为 5000 .

GA 优化计算的参数为: 种群规模 100 , 进化终 止代数 100 , 交叉概率 0.95 , 变异概率 $0.10, C_{\mathrm{scale}}=10^{7}$, $K=2.0$. 对所有节点取 $\left[P_{j}\right]=0.9,\left[R_{j}\right]=2$ 时, 优化计算得 到管网设计参数如图 12 所示.

\section{1 违约修补策略有效性验证}

采用 DCCG 01 初始种群, 分别利用 $\mathrm{T}_{1,3}, \mathrm{~T}_{1,3} \& \mathrm{~T}_{2}$, $\mathrm{T}_{1,2}\left(P_{\mathrm{L}}=0.5\right)$ 三种个体修补策略进行 10 次独立优化计
算, 得到的最优适应度值(Fit-max)、平均适应度值 (Fit-mean)、平均目标函数值(Obj-mean)、可行解空间 $\boldsymbol{\Omega}^{c}$ 的个体数量(Cons-num)共 4 种指标的均值统计结 果随进化代数的变化趋势见图 13 , 其中利用 $\mathrm{T}_{1,3} \& \mathrm{~T}_{2}$ 修补策略第 1 次优化计算过程中种群个体所处搜索 空间位置随进化代数变化趋势见图 14; 假定节点 11 , 17 为重要节点, 其度约束为 $\left[R_{11}\right]=\left[R_{17}\right]=3$, 其他节点
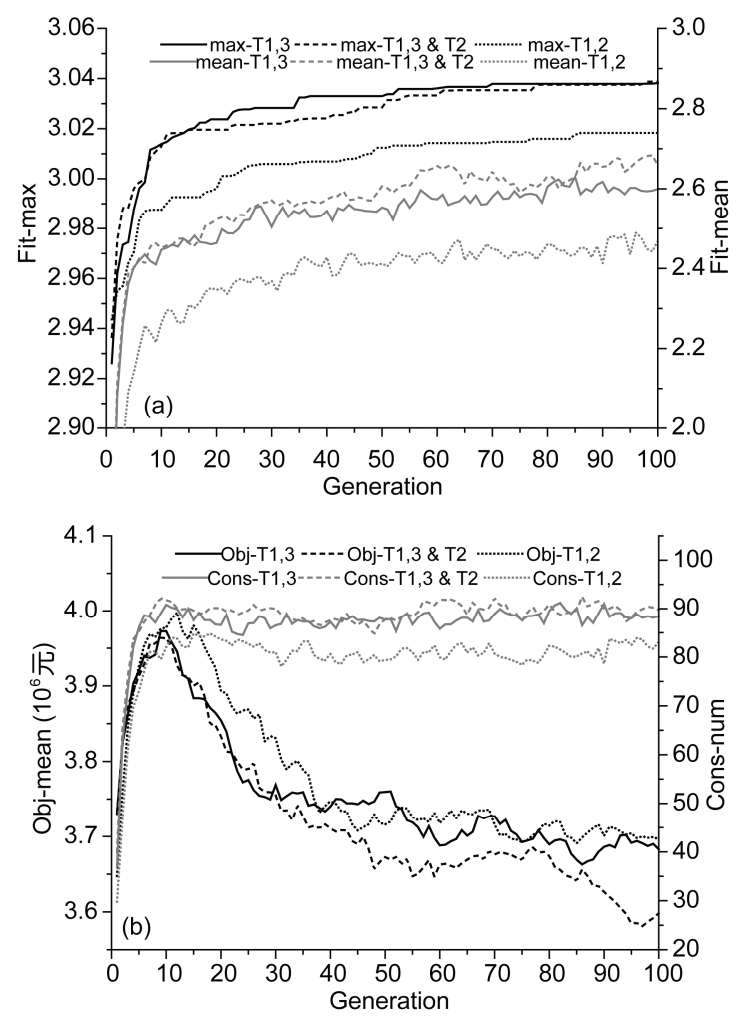

图 13 相同初始种群不同修补策略优化计算对比

(a) 适应度函数最大值与平均值; (b) 目标函数值与满足约束条件 个体数

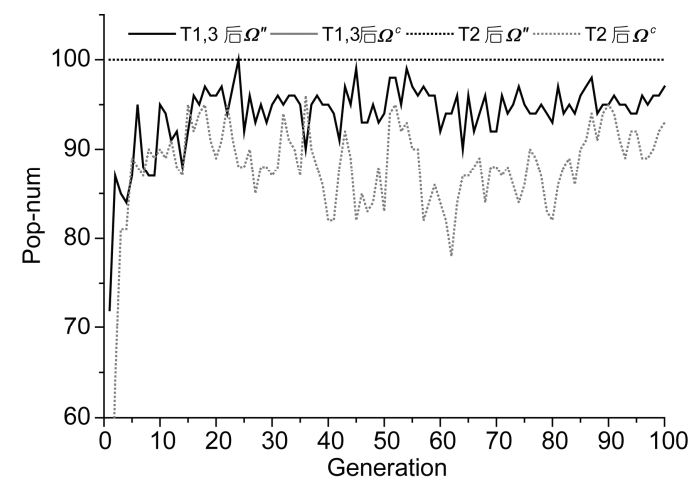

图 14 单次优化计算种群个体搜索空间分布统计 $\left(\mathbf{T}_{1,3} \& \mathbf{T}_{2}\right)$ 
$\left[R_{j}\right]=2$, 所有节点 $\left[P_{j}\right]=0.9$, 利用 $\mathrm{T}_{1,3}$ 和 $\mathrm{T}_{1,3} \& \mathrm{~T}_{2}$ 修补 策略的计算统计结果见图 15.

由图 13 可知, 对于 Fit-mean 及 Obj-mean 结果, 修补策略间的优劣关系为 $T_{1,3} \& T_{2}>T_{1,3}>T_{1,2}$; 而对于 Fit-max 及 Cons-num 结果, 相应的关系为 $T_{1,3} \& T_{2} \approx$ $\mathrm{T}_{1,3}>\mathrm{T}_{1,2}$; 由此可认为本文提出的修补策略 $\left(\mathrm{T}_{1,3}\right.$, $\mathrm{T}_{1,3} \& \mathrm{~T}_{2}$ )均优于文献[3]的修补策略.

图 14 中, $\mathrm{T}_{1,3}$ 修补后搜索空间内个体数量关系为 $\boldsymbol{\Omega}^{\prime \prime} \geqslant \boldsymbol{\Omega}^{c}$, 与图 3 及图 10(d)的分析结果一致; $\mathrm{T}_{2}$ 修补后 种群中所有个体(种群规模 100)均集中于 $\boldsymbol{\Omega}^{\prime \prime}$ 空间内, 与图 10(e)的分析结果一致; 对于 $\boldsymbol{\Omega}^{c}$ 空间内的个体数 存在关系 $\mathrm{T}_{2}$ 后 $>\mathrm{T}_{1,3}$ 后, 说明经过 $\mathrm{T}_{2}$ 修补后部分个体 从空间 $\boldsymbol{\Omega}^{\prime}$ 直接转换至 $\boldsymbol{\Omega}^{c}$ 空间内, 而不必经过 $\boldsymbol{\Omega}^{\prime \prime} \boldsymbol{\Omega}^{c}$ 空间，与图 10(e)的分析结果一致.

图 13 中部分指标反映出 $\mathrm{T}_{1,3} \& \mathrm{~T}_{2} \approx \mathrm{T}_{1,3}$, 未能完 全体现 $T_{2}$ 修补的优势, 当算例中增加部分节点度约 束时(图 15), 4 种指标的计算统计结果均存在 $T_{1,3} \& T_{2}$ 优于 $\mathrm{T}_{1,3}$, 从而表明 $\mathrm{T}_{2}$ 修补策略的有效性.
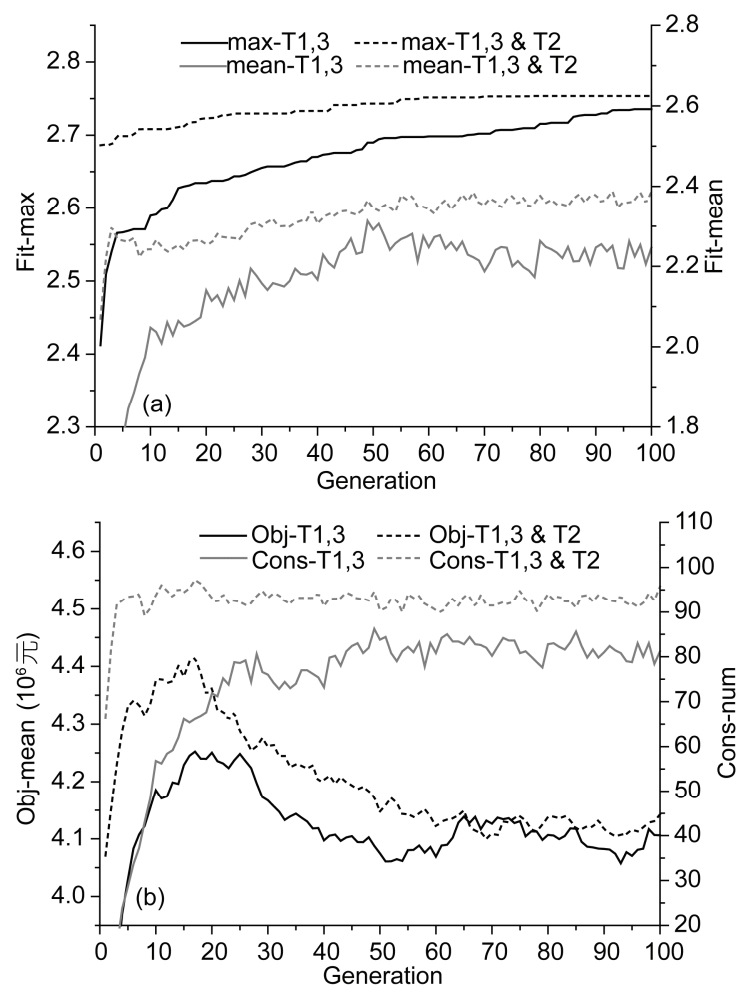

图 15 增加节点度约束后不同修补策略优化计算对比 (a) 适应度函数最大值与平均值; (b) 目标函数值与满足约束条件 个体数

\subsection{DCCG 初始种群有效性验证}

分别采用 DCCG 随机种群(-D)和随机初始种群 $(-\mathrm{R})$, 所有节点取 $\left[P_{j}\right]=0.9,\left[R_{j}\right]=2$ 时, 利用 $\mathrm{T}_{1,3}$ 和 $\mathrm{T}_{1,3} \& \mathrm{~T}_{2}$ 修补策略的 10 次独立计算统计平均结果见图 16.

由图 16 可知, Fit-max, Fit-mean 和 Obj-mean 3 个 指标, 对于 $\mathrm{T}_{1,3}$ 和 $\mathrm{T}_{1,3} \& \mathrm{~T}_{2}$ 均存在 DCCG 随机种群优 于随机种群; 虽然指标 Cons-num 的结果未能体现出 明显差异, 但由于 Fit 和 Obj 指标可以体现最终优化 计算的效率, 因此可以认为 DCCG 生成初始种群策 略优于随机生成初始种群的方法.

对比图 13 和 16 中 $\mathrm{T}_{1,3}, \mathrm{~T}_{1,3} \& \mathrm{~T}_{2}$ 的 Fit-mean 和 Obj-mean 指标的统计结果可知, DCCG 01 种群优于 DCCG 随机种群. 这是由于本文目标函数为 $\mathrm{min}$ 型, 且编码 3 对应的可靠度增加值的造价系数高于编码 1, 而 DCCG 随机种群中含有编码 3. 由编码规则可 知, 最优个体中基因位编码为 1 (抗震可靠度为 0.90 ) 的管段最多, 与图 12 所示的优化结果一致.
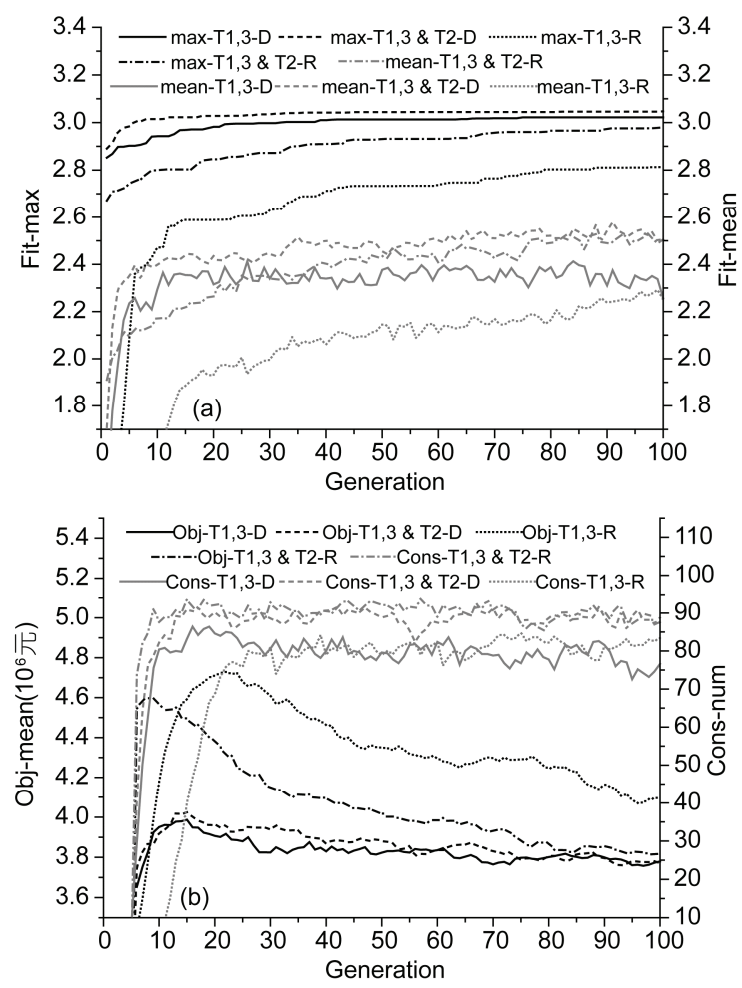

图 16 DCCG 随机种群与随机初始种群优化计算对比 (a) 适应度函数最大值与平均值; (b) 目标函数值与满足约束条件 个体数 


\section{3 相同耗时的计算效率比较}

图 13 中以 $T_{1,3} \& T_{2}$ 工况的 10 次平均计算时间为 基准计算时间(1.0)时, $\mathrm{T}_{1,2}$ 和 $\mathrm{T}_{1,3}$ 工况对应的平均计算 时间分别为 0.77 和 0.85 . 图 15 中以 $\mathrm{T}_{1,3} \& \mathrm{~T}_{2}$ 工况的 10 次平均计算时间为基准计算时间(1.0)时, $\mathrm{T}_{1,3}$ 工况 对应的平均计算时间为 0.70 . 图 16 中以 $T_{1,3} \& T_{2}-D$ 工 况的 10 次平均计算时间为基准计算时间(1.0)时, $\mathrm{T}_{1,3}-\mathrm{D}, \mathrm{T}_{1,3}-\mathrm{R}$ 和 $\mathrm{T}_{1,3} \& \mathrm{~T}_{2}-\mathrm{R}$ 修补策略对应的平均计算 时间分别为 $0.91,0.98$ 和 $0.9957(\approx 1.0)$.

为比较相等计算时间内不同工况的计算结果, 将优化计算的耗时对所有进化代数 (100 代)做平均; 图 13 中, 将 $T_{1,3} \& T_{2}$ 工况第 77,85 代的结果与 $T_{1,2}, T_{1,3}$ 工况第 100 代的结果进行比较, 比较近似相等计算耗 时的计算结果, 相应结果见表 1 (图 15 和 16 的计算时 间比较也采用此方法). 从表 1 第 1,3 和第 2, 4 行的结 果比较可知, 在近似相等计算时间内 $T_{1,3} \& T_{2}$ 工况的 计算结果优于 $\mathrm{T}_{1,2}$ 和 $\mathrm{T}_{1,3}$ 工况; 对第 6,7 行的结果比 较可知, $\mathrm{T}_{1,3} \& \mathrm{~T}_{2}$ 工况的计算结果优于 $\mathrm{T}_{1,3}$ 工况; 对第 9, 11 行的结果比较可知, $\mathrm{T}_{1,3} \& \mathrm{~T}_{2}-\mathrm{D}$ 工况的计算结果 优于 $\mathrm{T}_{1,3}-\mathrm{D}$ 工况; 对第 10,13 行的结果比较可知, $\mathrm{T}_{1,3} \& \mathrm{~T}_{2}-\mathrm{R}$ 工况的计算结果优于 $\mathrm{T}_{1,3}-\mathrm{R}$ 工况; 对第 10 , 13 行的结果比较可知, $\mathrm{T}_{1,3} \& \mathrm{~T}_{2}-\mathrm{R}$ 工况的计算结果优 于 $\mathrm{T}_{1,3}-\mathrm{R}$ 工况.

需要说明的是, 上述工况的计算时间比较, 仅是 优化计算过程的耗时比较, 并未考虑 DCCG 初始种
群生成所需的时间. 对于图 16 工况对应的问题, 采 用标准 GA 算法求解(2)式模型(种群规模 1000 , 进化 终止代数 100 , 交叉概率 0.95 , 变异概率 0.10 )得到 DCCG 01 种群的计算时间约为 $\mathrm{T}_{1,3} \& \mathrm{~T}_{2}$-D 工况基准计 算时间的 0.33 倍，若考虑到 10 次计算均采用同一 DCCG 01 种群生成 DCCG 随机种群, 则生成 DCCG 初始种群在每次优化计算中增加的时间仅为 0.033 倍 基准时间，可以认为其对总计算时间的影响较小.

\section{6 结论}

城市供水管网抗震设计模型, 以用户节点的抗 震连通可靠度和节点连接度作为设计指标, 以管网 拓扑结构和管段抗震能力为设计参数. 寻找最优设 计参数为离散变量组合优化问题, 由于无法建立个 体编码与可行解的唯一映射关系, 约束条件的存在 使编码空间大于可行解空间.

根据模型特点、约束条件与优化搜索空间的关系, 首先利用 DCCG 生成初始种群, 缩小了初始搜索空 间; 然后针对优化过程中产生的违约个体会扩大搜 索空间的问题，根据约束条件的特点提出了两种递 进型的违约个修补策略，缩小优化过程中的搜索空 间, 实现了随进化代数动态缩减搜索空间. 方法对比 及算例分析表明, 本文提出的两阶段搜索空间缩减 策略有良好的求解效率, 也可为其他有约束离散变 量优化计算问题提供参考.

表 1 相等计算时间下不同工况计算结果对比

\begin{tabular}{|c|c|c|c|c|c|c|c|}
\hline 图名 & 代数 & 工况 & 行号 & Fit-max & Fit-mean & Obj-mean $\left(10^{6}\right.$ 元 $)$ & Cons-num \\
\hline \multirow{5}{*}{13} & 77 & $\mathrm{~T}_{1,3} \& \mathrm{~T}_{2}$ & 1 & 3.0353 & 2.6117 & 3.6850 & 90.4 \\
\hline & 85 & $\mathrm{~T}_{1,3} \& \mathrm{~T}_{2}$ & 2 & 3.0375 & 2.6300 & 3.6411 & 89.6 \\
\hline & \multirow{3}{*}{100} & $\mathrm{~T}_{1,2}$ & 3 & 3.0183 & 2.4678 & 3.6962 & 83.1 \\
\hline & & $\mathrm{T}_{1,3}$ & 4 & 3.0382 & 2.5992 & 3.6827 & 88.4 \\
\hline & & $\mathrm{T}_{1,3} \& \mathrm{~T}_{2}$ & 5 & 3.0388 & 2.6589 & 3.5982 & 89.7 \\
\hline \multirow{3}{*}{15} & 70 & $\mathrm{~T}_{1,3} \& \mathrm{~T}_{2}$ & 6 & 2.7531 & 2.3537 & 4.1097 & 91.4 \\
\hline & \multirow{2}{*}{100} & $\mathrm{~T}_{1,3}$ & 7 & 2.7357 & 2.2463 & 4.1060 & 81.2 \\
\hline & & $\mathrm{T}_{1,3} \& \mathrm{~T}_{2}$ & 8 & 2.7537 & 2.3861 & 4.1422 & 95.8 \\
\hline \multirow{6}{*}{16} & 91 & $\mathrm{~T}_{1,3} \& \mathrm{~T}_{2}-\mathrm{D}$ & 9 & 3.0459 & 2.5787 & 3.7826 & 86.8 \\
\hline & 98 & $\mathrm{~T}_{1,3} \& \mathrm{~T}_{2}-\mathrm{R}$ & 10 & 2.9758 & 2.5111 & 3.8168 & 90.0 \\
\hline & \multirow{4}{*}{100} & $\mathrm{~T}_{1,3}-\mathrm{D}$ & 11 & 3.0218 & 2.2560 & 3.7793 & 76.6 \\
\hline & & $\mathrm{T}_{1,3} \& \mathrm{~T}_{2}-\mathrm{D}$ & 12 & 3.0474 & 2.5132 & 3.7711 & 88.8 \\
\hline & & $\mathrm{T}_{1,3}-\mathrm{R}$ & 13 & 2.8136 & 2.2514 & 4.1000 & 83.4 \\
\hline & & $\mathrm{T}_{1,3} \& \mathrm{~T}_{2}-\mathrm{R}$ & 14 & 2.9806 & 2.5053 & 3.8218 & 87.6 \\
\hline
\end{tabular}


参考文献

1 Sevtap-Selcuk A, Semih-Yucemen M. Reliability of lifeline networks under seismic hazard. Reliab Eng Syst Safe, 1999, 65: 213-227

2 中华人民共和国建设部. 城市抗震防灾规划标准(GB50413-2007). 北京：中国建筑工业出版社, 2007

3 李杰, 邢燕. 基于可靠度的生命线工程网络抗震设计. 同济大学学报(自然科学版), 2010, 38: 783-786

4 李杰. 生命线工程抗震一基础理论与应用. 北京: 科学出版社, 2005

5 陈玲俐. 城市供水管网系统抗震功能可靠性分析与优化. 博士学位论文. 上海: 同济大学, 2002

6 Kadu M S, Gupta R, Bhave P R. Optimal design of water networks using a modified genetic algorithm with reduction in search space. J Water Res Plan Man, 2008, 134: 147-160

7 Tolson B A, Maier H R, Simpson A R, et al. Genetic algorithms for reliability-based optimization of water distribution systems. J Water Res Plan Man, 2004, 130: 63-72

8 庄宝玉，杨宇飞，赵新华. 基于改进混合蛙跳算法的供水管网优化. 中国给水排水, 2011, 27: 45-49

9 刘威, 徐良, 李杰. 供水管网抗震拓扑优化算法研究. 中国科学.技术科学, 2012, 42: 1351-1360

10 唐世浩, 朱启疆. 遗传算法中初始种群与交叉、变异率对解的影响及其解决方案. 科技通报, 2001, 17: 1-7

11 Leung Y W, Wang Y. An orthogonal genetic algorithm with quantization for global numerical optimization. IEEE Trans Evol Comput, 2001, 5: $41-53$

12 何大阔, 王福利, 贾明兴. 遗传算法初始种群与操作参数的均匀设计. 东北大学学报, 2005, 26: 828-831

13 张建勇, 李军. 具有同时配送和回收需求的车辆路径问题的混合遗传算法. 中国公路学报, 2006, 19: 118-122

14 Coello Coello C A. Theoretical and numerical constraint-handling techniques used with evolutionary algorithms: A survey of the state of the art. Comput Meth Appl Mech Eng, 2002, 191: 1245-1287

15 Chootinan P, Chen A. Constraint handling in genetic algorithms using a gradient-based repair method. Comput Oper Res, 2006, 33: 2263-2281

16 Koziel S, Michalewicz Z. Evolutionary algorithms, homomorphous mappings, and constrained parameter optimization. Evol Comput, 1999, 7: $19-44$

17 Reichelt D, Rothlauf F, Gmilkowsky P. Designing reliable communication networks with a genetic algorithm using a repair heuristic. In: Gottlieb J, Raidl G R, eds. Evolutionary Computation in Combinatorial Optimization. Lecture Notes in Computer Science, Vol 3004. Berlin, Heidelberg: Springer, 2004. 177-187

18 Altiparmak F, Dengiz B. A cross entropy approach to design of reliable networks. Eur J Oper Res, 2009, 199: 542-552

19 马良, 蒋馥. 度约束最小生成树的快速算法. 运筹与管理, 1998, 7: 1-5

20 王励成, 孙麟平. 求解度限制最小生成树问题的启发式遗传搜索算法. 系统工程理论与实践, 2003, 5: 103-107

21 包元锋, 李杰. 基于遗传算法的生命线工程网络抗震优化设计. 防灾减灾工程学报, 2006, 26: 21-27

\title{
A heuristic search space reduction method for water distribution system seismic design optimization
}

\author{
HOU BenWei \& DU XiuLi
}

Key Laboratory of Urban Security and Disaster Engineering of Ministry of Education, Beijing University of Technology, Beijing 100124, China

In the seismic design optimization model of urban water distribution system (WDS), the design objective is seismic performance requirement of user nodes, the design parameters consist of the water supply network topology and the seismic capacity of pipeline structures. The selection of optimal design parameters is a combinatorial optimization problem. This paper presents a two-stage search space reduction strategy for WDS seismic design using GA. In the first stage, the initial population is generated by degree constrained connected graph, which narrowed the optimization search space at the beginning of GA. A genetic individual repair strategy is proposed for individuals violate constraints in the evolutionary process, which achieved search space reduction during GA operation. The proposed method processes better efficiency in WDS seismic optimized design using GA and also provides a reference for other constrained optimization problems.

water distribution system, seismic design optimization, constrained optimization of discrete variables, search space reduction doi: 10.1360/N092014-00235 